\title{
La motiline. De l'animal à l'homme
}

La motiline est l'un des nombreux peptides de la muqueuse intestinale. A la suite d'expériences effectuées sur des animaux, on a pu associer les variations de la motilinémie à la régulation de la motricité gastrointestinale interdigestive au moment où se déclenche la phase III du complexe moteur migrant. L'action de la motiline dans la physiologie et dans la pathophysiologie humaine n'est pas encore très précise ; son intérêt clinique, cependant, semble s'affirmer de plus en plus. En effet, la stimulation des récepteurs de la motiline qui se trouvent dans l'antre gastrique entraîne de très puissantes contractions de cette partie de l'estomac ; on peut en déduire que la motiline (ou des molécules dérivées de la motiline) pourrait dans un avenir très proche servir au traitement de la gastroparésie.

Pierre Poitras, Michel Boivin, Serge St-Pierre

\footnotetext{
ADRESSE

P. Poitras : professeur agrégé. Michel Boivin : adjoint de recherche, département de médecine, Université de Montréal. Centre de recherche clinique André-Viallet. Hôpital St-Luc, 1058, rue St-Denis, Montréal, Québec, H2X 3J4 Canada.

Serge St-Pierre: professeur, INRS-Santé, Université du Québec, 245, boulevard Hymus, Pointe-Claire.

\section{Introduction}

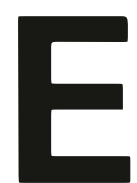

n 1972, J.C. Brown a extrait puis purifié, de la muqueuse intestinale porcine, un peptide composé de 22 acides aminés qui avait la propriété de stimuler l'activité motrice de l'estomac : il l'appcla motiline [1]. Depuis lors, des chercheurs ont pu évaluer l'action de la motiline chez de nombreuses espèces animales. Les expériences effectuées sur le chien ont le plus contribué à comprendre les mécanismes d'action de ce peptide. Il scmble que les travaux de physiologie animale et humaine puissent déboucher maintenant sur des applications d'ordre pratique pour le médecin clinicien ; l'intérêt de ces applications tient surtout au potentiel thérapeutique de la motiline ou de ses analogues. Nous allons passer en revue les connaissances acquises concernant l'action et le rôle de la motiline chez les mammi- fères et tout particulièrement chez l'homme.

\section{Structure moléculaire}

La difficulté a reconnaître par différents anticorps les motilines présentes chez différentes espèces animales suggérait une importante hétérogénéité structurale selon les espèces. Nous avons publié, en 1983, la structure de la motiline canine [2]. Tout comme la motiline porcine, le peptide extrait de la muqueuse intestinale du chien puis purifié était constitué de 22 acides aminés. Cependant, seuls 17 des 22 résidus sont communs aux deux molécules (voir figure 1). Quelques années plus tard, grâce aux techniques de biologie moléculaire, on a reconnu que les motilines porcine et humaine comportaient une séquence identique d'acides aminés [3]. Plus récemment, la structure de la motiline du lapin [4, 5] ainsi que du chat (T.L. Peeters, commu- 


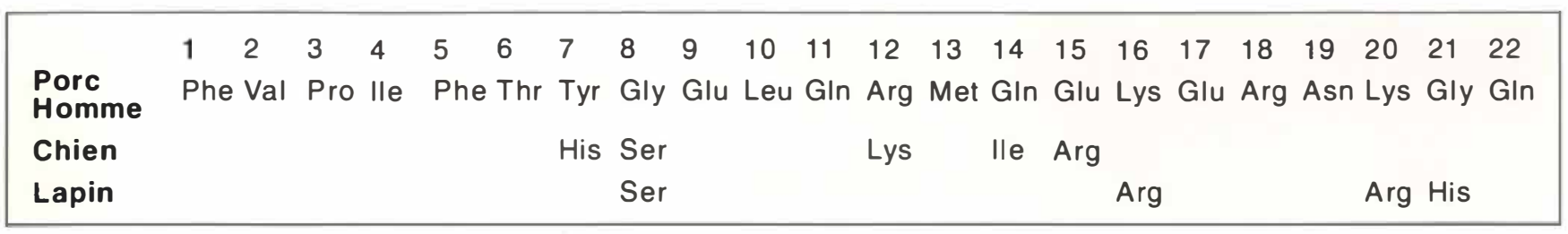

Figure 1. Séquence des acides aminés de la motiline retrouvée chez différentes espèces animales.

nication personnelle) fut reconnue, alors que celle du rat demeure toujours une enigme.

A l'aide de la spectroscopie par résonnance magnétique nucléaire, on a pu analyser la structure tertiaire de la motiline. Les 6 premiers acides aminés apparaissent repliés autour d'une large boucle ; de la position 9 à la position 20 on retrouve une formation helicoïdale alpha [6].

En dépit des différences de structures, la plupart des analyses expérimentales ont montré que l'activité biologique de la motiline canine et de la motiline porcine, ou humaine, étaient comparables [7]. Le phénomène s'explique facilement car la motiline des deux espèces comprend une structure moléculaire identique située dans la portion $\mathrm{N}$-terminale de la molécule et responsable de son activité biologique [8].

Tant chez l'homme que chez l'animal (dans la circulation périphérique aussi bien que dans la muqueuse intestinale) prédomine une forme moléculaire : la motiline 1-22 [3, 9]. Chez l'homme existe une prépromotiline de 114 acides aminés, constituée d'un peptide signal formé de 25 résidus, suivi du peptide original (motiline 1-22) qui précède une séquence de 65 acides aminés (que nous avons appelée MRP (Motilin Related Peptide)) [3]. On n'a pu pour l'instant associer le peptide MRP à aucune autre substance existante; on ne l'a pas non plus retrouvé dans l'intestin ou le plasma de l'homme ou du chien; on ignore donc son intérêt sur le plan biologique. Le gène de la motiline humaine a été repéré sur le chromosome $6[10]$. La structure génomique de la motiline est particulière ; en effet elle est constituée de 5 exons séparés par 4 introns, les exons 2 et 3 constituant le peptide bioactif $[11,12]$.

\section{Localisation}

mal la motiline a été localisée presqu'uniquement dans la muqueuse digestive [9]. On a reconnu la cellule endocrine synthétisant la motiline (la cellule M) chez l'homme et chez le chien au sein de la muqueuse de l'intestin grêle proximal [12]. Certains travaux auraient permis de détecter la présence de motiline dans certaines structures extradigestives, comme le système nerveux central ou périphérique. Cependant, les quantités souvent minimes de motiline et l'incertitude quant à la nature du produit décelé mettent en doute le rôle de la motiline comme agent neurotransmetteur [13]. Toutefois, l'influence que pourrait exercer la motiline sur certaines fonctions cérébrales, comme la sécrétion d'hormone de croissance [14], plaide en faveur d'une action neurocrine qui demeure toujours à préciser.

\section{Activité biologique, récepteur et mode d'action}

L'activité biologique prépondérante de la motiline c'est la stimulation de la contraction du muscle lisse intestinal. L'injection intraveineuse de motiline produit chez les mammiferes des contractions du sphincter œsophagien inférieur, de l'antre stomacal et du duodénum (figure 2). Ces contractions se propagent ensuite le long de l'intestin grêle. Le profil de contraction que l'on enregistre après la perfusion de motiline est semblable à celui que l'on observe lors de la phase III du complexe moteur migrant (CMM). La phase III est une puissante onde péristaltique qui parcourt l'appareil digestif de l'estomac au colon selon un cycle de 90 à $100 \mathrm{~min}$. lors de la période interdigestive de nombreux mammiferes et, notamment, l'homme et le chien. La motiline peut aussi provoquer de faibles contractions sigmoïdiennes [15]. Chez le chien, mais non chez l'homme, l'injection de motiline pourra aussi susciter la contraction de la vésicule biliaire. Enfin, la motiline s'avère un faible stimulant des fonctions sécrétrices, tant celles de l'estomac que celles du pancréas [16].

On ignore encore qu'elle est la structure moléculaire du récepteur de motiline. Les résultats des expériences effectuées sur le chien laissent supposer que ce récepteur ne se trouve pas à la surface de la cellule musculaire ; ainsi, l'action de la motiline sur la contraction du muscle lisse intestinale s'exercerait-elle par l'intermédiaire des structures nerveuses propres à la paroi intestinale [17]. A cet égard, si le rôle de l'acétylcholine à titre de médiateur de l'activité contractile de la motiline est évident, celui d'autres agents comme la sérotonine, les opiacés endogènes, le Polypeptide Vasolntestinal (VIP) et l'oxyde d'azote (NO) [18] n'est sûrement pas négligeable. Chez l'homme, par contre, les résultats obtenus à l'aide de divers modèles expérimentaux nous donnent fortement à penser que des récepteurs spécifiques de la motiline se trouvent bel et bien sur la membrane du muscle lisse intestinal [19].

Récemment, on a démontré que l'érythromycine et certains dérivés de l'érythromycine pouvaient stimuler l'activité motrice intestinale grâce à leur capacité de liaison avec les récepteurs de la motiline [20]. Cependant, le phénomène qui expliquerait la liaison de l'érythromycine avec les récepteurs de la motiline reste encore à découvrir.

\section{La motilinémie et sa régulation}

Le profil que dessinent les variations des concentrations plasmatiques de la motiline décrit une situation singulière. En période de jeûne, la motiline est secrétée de façon irrégulière dans la circulation périphérique. On 
a en effet remarqué des élévations brèves de la motilinémie ; ces élévations sont répétitives et cycliques, et elles persistent tout au long de la période interdigestive $[21,22]$. Chez le chien, on a pu étudier la régulation de la libération intermittente de motiline dans la circulation périphérique. Ce phénomène ne semble pas mettre en jeu de façon significative le système nerveux sympathique; en revanche, certains indices permettraient de reconnaître au nerf vague une fonction inhibitrice. Cependant, le rôle précis de ce nerf demeure encore obscur puisque les animaux vagotomisés présentent un cycle de sécrétion de motiline normal [23]. L'injection d'atropine ou de somatostatine [21] diminue la motilinémie ; par contre, la bombésine [24] ou les agonistes opiacés [25] augmentent les taux de la motiline dans la circulation sanguine. Les résultats d'expériences faites in vitro à l'aide de cellules isolées de la muqueuse intesti- nale canine révèlent la présence de récepteurs muscariniques sur la membrane de la cellule qui sécrète la motiline et suggèrent donc que l'acétylcholine occupe une place prépondérante dans la stimulation de la cellule à motiline [26].

On retrouve également le cycle de variation de la motilinémie chez l'homme en période de jeûne [22]. En période postprandiale le profil de la motilinémie chez l'homme est très différent de celui obtenu chez le chien. Chez le chien, l'augmentation cyclique de la motilinémie de jeûne est abolie par l'ingestion d'un repas [21]. Chez l'homme le fait de manger entraîne, au contraire, une augmentation de la motilinémie (voir figure 3 ). Cette augmentation survient dans les 30 premières minutes suivant l'ingestion du repas et elle atteint les niveaux de concentrations plasmatiques observés en période interdigestive lors des pics de motilinémie associés à la phase III du complexe moteur migrant (CMM) [22]. Pour tenter d'expliquer une augmentation aussi rapide de la motilinénie lors d'un repas, nous avons récemment démontré que la stimulation céphalique obtenue par la prise d'un repas fantôme (sham feeding), tout comme la distension gastrique réalisée à l'aide d'un ballon gonflable, pouvaient entraîner une élévation de la motilinémie [27].

\section{Rôle physiologique}

a) Action interdigestive

Le rôle physiologique de la motiline est maintenant clairement établi chez le chien. On peut actuellement conclure que la motiline, grâce à la fluctuation de ses concentrations plasmatiques durant la période de jeûne, participe au déclenchement de la phase III du CMM. Voici les principales données mises en évidence par les travaux de recherche expérimentale :

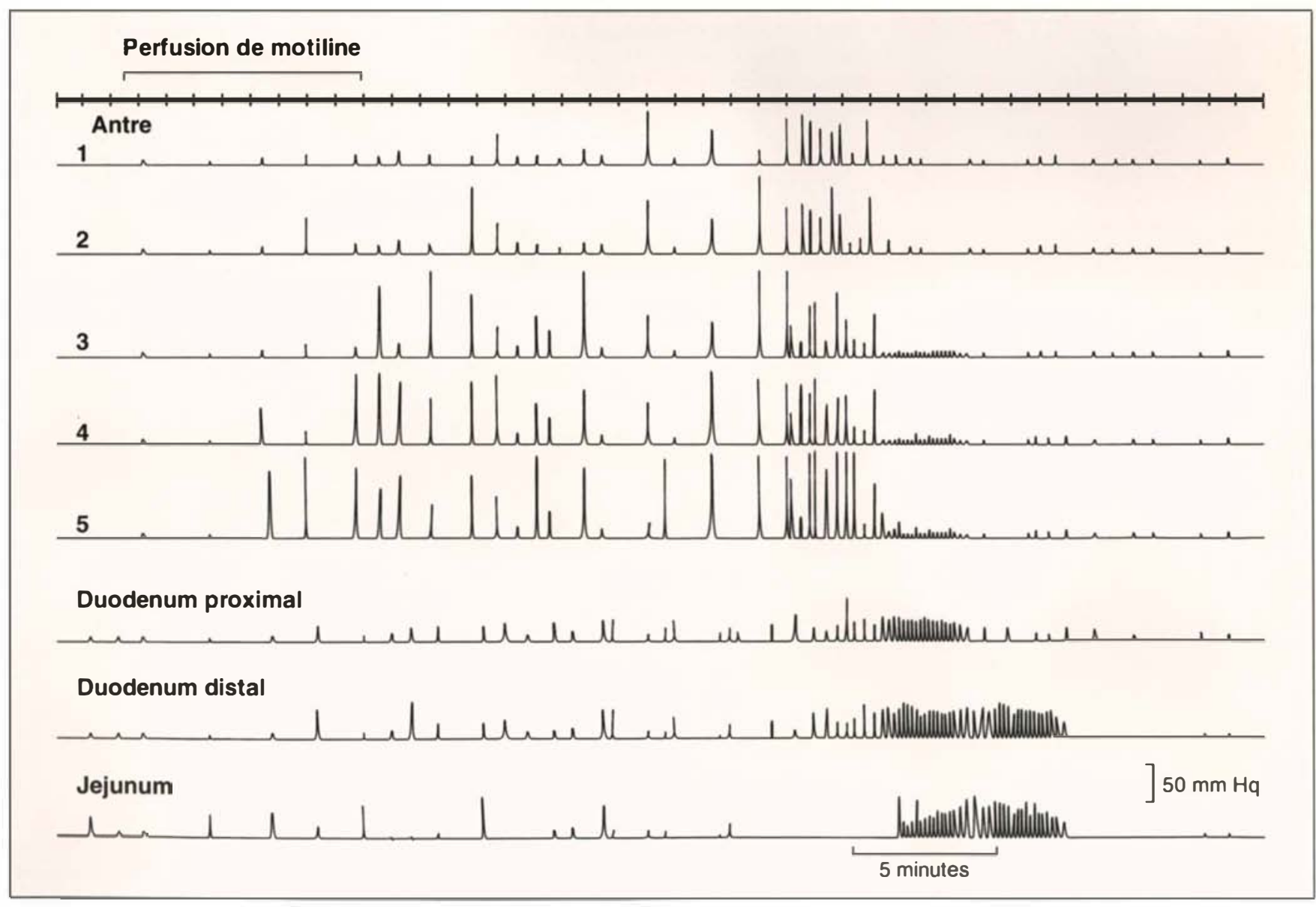

Figure 2. Contraction intestinale péristaltique induite par la perfusion de motiline (25ng Kg') chez l'homme. Le profil de contraction mime celui de la phase III du complexe moteur migrant (CMM) interdigestif. 


\section{RÉFÉRENCES}

1. Brown JC, Cook MA, Dryburgh JR. Motilin, a gastric motor activity-stimulating polypeptide : final purification, amino acid composition, and c-terminal residues. Gastrœenterology $1972 ; 62: 401-4$.

2. Poitras P, Reeve JR Jr, Hunkapiller MW, Hood LE, Walsh JH. Purification and characterization of canine intestinal motilin. Reg Peptides 1983 ; 5 : 197-208

3. Dea D, Boileau G, Poitras P Lahaie RG. Molecular heterogeneity of human motilin-like immunoreactivity explained by the processing of prepromotilin. Gastroenterology $1989 ; 96: 695-703$.

4. Peeters T.L., Gossen D., Depoortere I., Vandermeers A., Christophe J., Vantrappen G. Isolation and characterization of rabbit motilin. J Gastroint Motility 1991 ; 3 : 66 .

5. McIntosh C.H.S., Mac Gillivray R.T.A., Brown J.C., Banfield D.F. Isolation and characterization of rabbit motilin precursor cDNA. Reg Peptides 1992; 40 : 206

6. Edmonson S., Schriver J., Zdunre J., Graslund A. The solution structure of motilin from NMR distance constraints, distance geometry, molecular dynamics and iterative full relaxation matrix refinement. Biochem $1990 ; 30$ : 11271-9.

7. Poitras P, Lahaie RG, St-Pierre S, Trudel L. Comparative stimulation of motilin duodenal receptor by porcine or canine motilin. Gastroenterology 1987 ; 92 : 658-62.

8. Poitras P, Gagnon D, St-Pierre S. Nterminal portion of motilin determines its biological activity. BBRC $1992 ; 183: 36-40$

9. Poitras P, Trudel L, Lahaie RG, Pomier-Layrargues G. Motilin-likeimmunoreactivity in intestine and brain of dog. Life Sciences 1987; 40 : 1391-5.

10. Daikh DI, Douglass JO, Adelman JP Structure and expression of the human motilin gene. DNA $1989 ; 8: 615-21$.

11. Yano H, Seino Y, Fujita J. Exon-intron organization, expression, and chromosomal localization of the human motilin gene. FEBS Letters $1989 ; 2$ : 248-52.

12. Usellini L, Buchan AMJ, Polak JM, Capella C, Cornaggia M, Solcia E. Ultrastructural localization of motilin in endocrine cells of human and dog intestine by the immunogold technique. Histochemistry 1984 ; 81 : 363-8.

13. Nilaver G, Beinfeld MC, Bond CT, Daikh D, Godfrey B, Adelman JP. Heterogeneity of motilin immunoreactivity in mammalian tissue. Synapse $1988 ; 2$ : 266-75

14. Samson WK, Lumpkin MD, Nilaver G, McCann SM. Motilin : a novel growth hormone releasing agent. Brain Res Bulletin $1984 ; 12,57-62$.

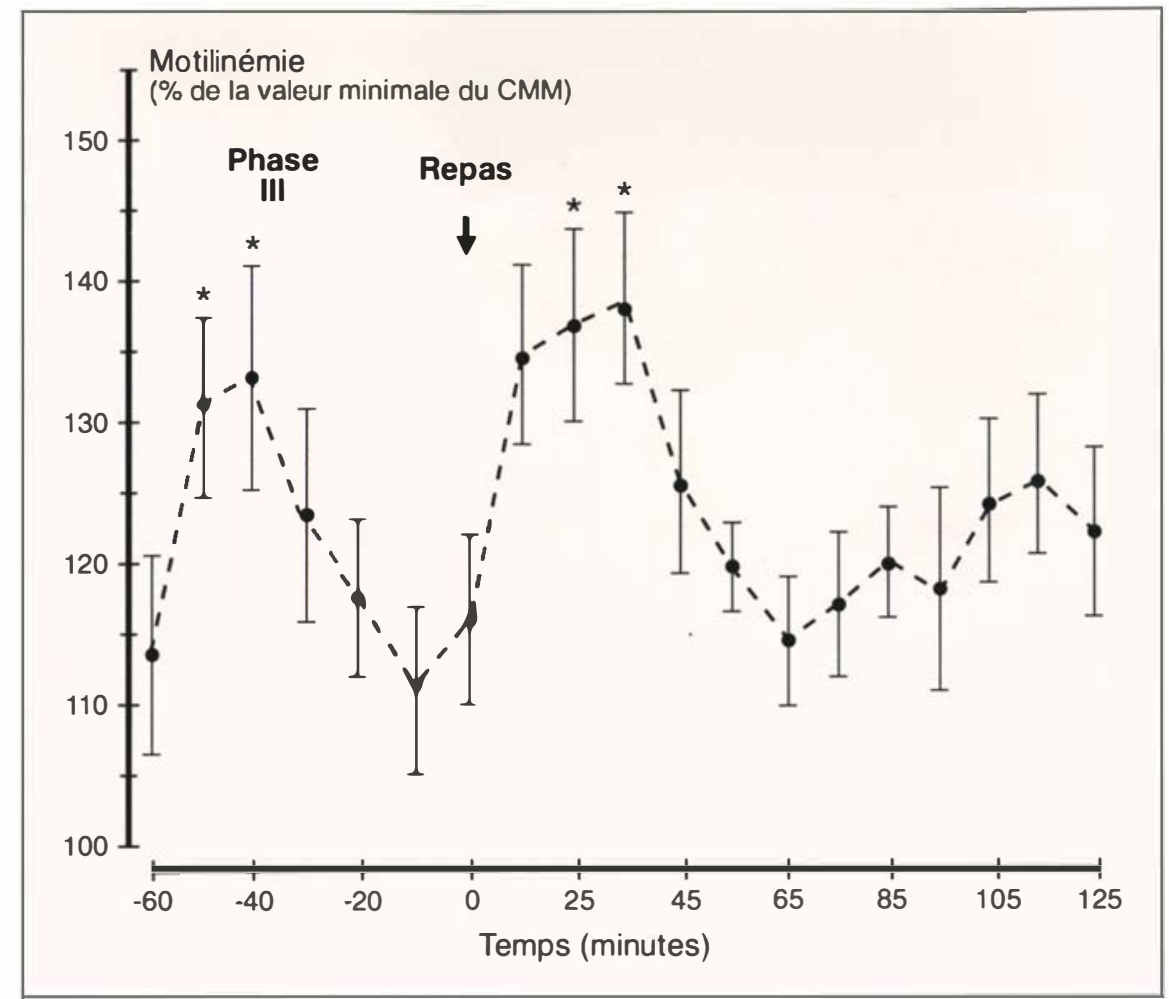

Figure 3. Augmentation de la motilinémie lors de l'ingestion d'un repas $1511 \mathrm{Kcal}$; 56 \% HC, 20 \% P, 20 \% G) chez I'homme (n=9). Les astériques indiquent des valeurs significativement supérieures $(p<0.05)$ aux valeurs minimales obtenues aux temps $-60,-10,55,65,75$ et 85 minutes.

1) Dès 1926, Farrel et Ivy [28] ont démontré qu'une portion de l'estomac canin transplantée dans la glande mammaire conservait une motricité identique à celle de l'estomac dont elle provenait, tant en période de jeûne qu'en période postprandiale. Par la suite, de nombreux auteurs, à l'aide de modèles expérimentaux différents, ont démontré que l'activité motrice de l'estomac était modulée par des facteurs circulants.

2) L'administration intraveineuse de motiline, à des doses reproduisant dans la circulation sanguine périphérique une augmentation physiologique de la motilinémie, produit sur le tube digestif supérieur des contractions péristaltiques similaires à celles observées durant la phase III du CMM [29].

3) Au cours de la période de jeûne, les élévations brèves, cycliques et régulières de la motilinémie, coïncident avec le début des phases III du tube digestif supérieur [21].

4) L'administration intraveineuse d'anticorps anti-motiline a fait dispa- raître du tube digestif proximal les contractions de la phase III [29].

On peut donc pratiquement certifier que la motiline circulante est un peptide dont l'action physiologique consiste à réguler la contraction du tube digestif proximal au cours de la période interdigestive chez le chien. L'activité contractile irrégulière et cyclique de l'intestin en période de jeûne s'accompagne de fluctuations de l'activité sécrétoire de divers organes digestifs. La participation de la motilinémie au contrôle de la sécrétion pancréatique interdigestive est bien documentée [30].

Chez l'homme, le rôle physiologique de la motiline demeure encore discuté. Il fut évidemment impossible de reproduire chez l'homme l'évidence expérimentale obtenue chez l'animal et démontrant la régulation hormonale de l'activité motrice gastrointestinale. Par ailleurs, tout comme chez le chien, les concentrations plasmatiques de motiline varient durant la période de jeûne, et il appert que les pics de motilinémie sont associés aux 


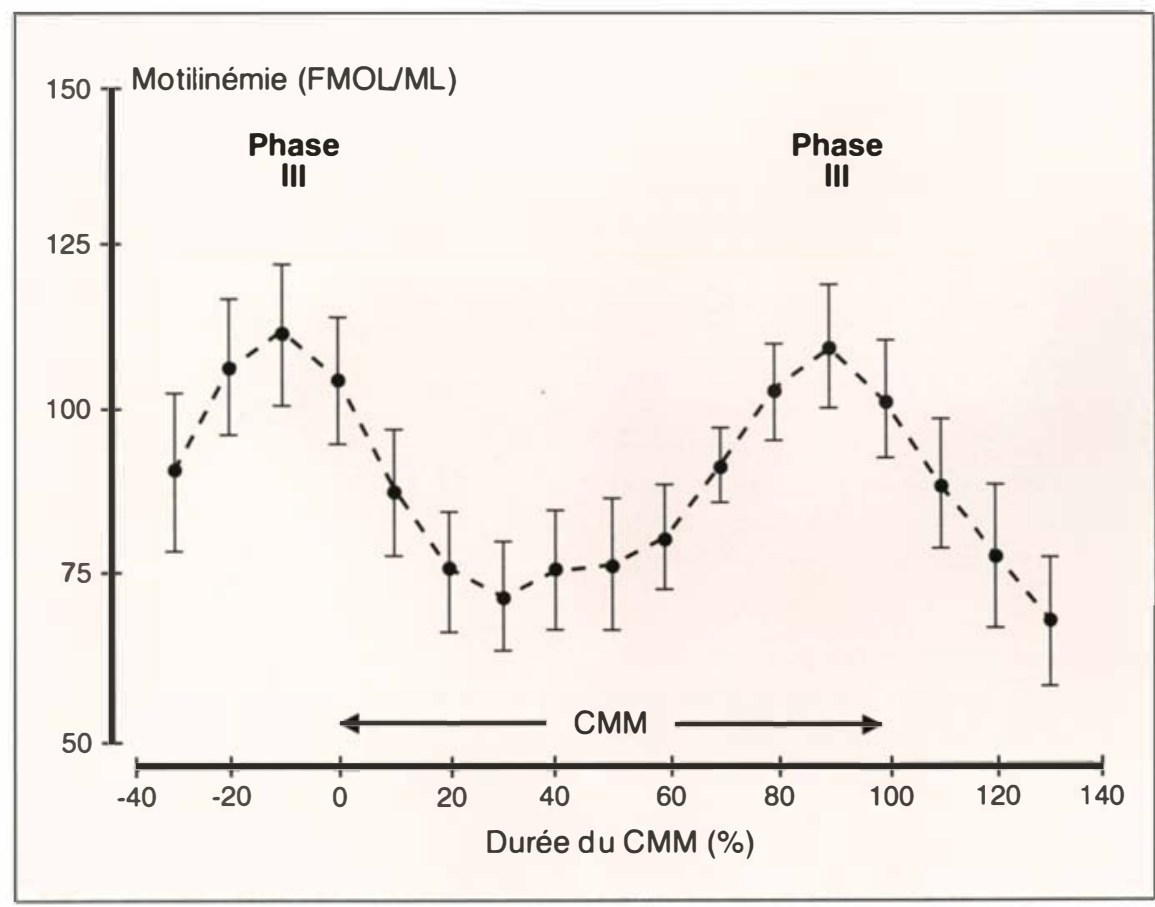

Figure 4. Variation de la motilinémie et activité motrice antrale en période de jeûne chez l'homme. Les pics de motiline sont couplés à la phase III du complexe moteur migrant (CMM). $(n=6)$.

phases III du CMM (figure 4). Ces pics de motilinémie sont cependant associés très spécifiquement aux phases III provenant de la région antrale et non à celles qui commencent après le pylore [22] (figure 5). L'injection de motiline chez l'homme déclenche (figure 2) une contraction antrale typique de la phase III qui se propage ensuite le long du tube digestif [22]. Cependant, il faut admettre qu'il nous fut impossible d'obtenir des résultats aussi probants que ceux enregistrés chez le chien en ce qui concerne l'effet de doses physiologiques de motiline exogène. Pour cerner l'activité physiologique de la motiline chez l'humain, les expériences sont difficiles à réaliser puisqu'il faut tenir compte de nombreux facteurs qui entrent en jeu comme, par exemple, la mesure par essai radioimmunologique de la motiline dans le plasma humain et l'enregistrement de la motricité antrale ; de plus la fiabilité de ces expériences dites physiologiques obtenues par des moyens malheureusement physiquement invasifs chez des individus donc soumis à un stress peut être mise en doute. La preuve ultime de la régulation du complexe migrant interdigestif par la motilinémie chez l'humain ne pourra être obtenue que lorsque des expériences pourront être réalisées à l'aide de substances synthétiques neutralisant de façon spécifique l'action de la motiline circulante.

b) Action digestive postprandiale
Chez le chien, l'activité postprandiale de la motiline semble nulle. En effet, après l'ingestion d'un repas, la libération cyclique de motiline s'interrompt [21]. De plus, l'administration de motiline, ou d'érythromycine agissant sur les récepteurs de la motiline, ne modifie jamais la motricité digestive postprandiale [31].

L'activité biologique reliée à l'hypermotilinémie postprandiale telle qu'on la rapporte chez l'homme demeure une énigme actuellement. Contrairement à la situation prévalant chez l'animal, les récepteurs de la motiline peuvent être activés lors de la période postprandiale chez l'homme. En effet, l'injection d'érythromycine, tant chez le sujet normal $[32,33]$ que chez le diabétique atteint de gastroparésie [34], pourra susciter de fortes contractions gastriques. Cependant, lorsque nous avons administré une dose de motiline légèrement supraphysiologique, nous n'avons pu observer de modification de la contractilité antrale telle qu'elle est normalement décelable après un repas, et par conséquent il apparaît fort peu probable que la motricité antrale puisse être modifiée par l'hypermotilinémie postprandiale normale [33]. A la suite de la découverte récente de récepteurs de la motiline situés dans le colon, nous

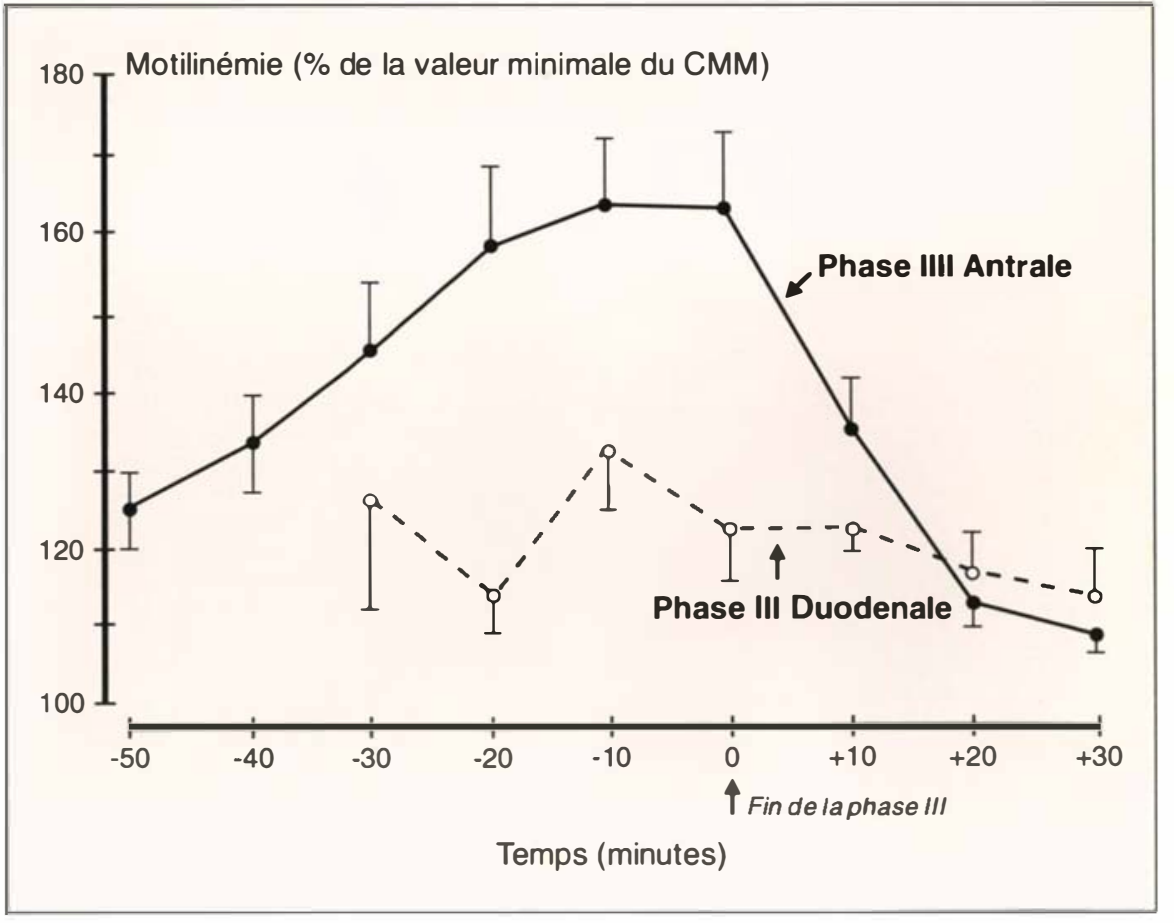

Figure 5. Variation de la motilinémie lors de phases III provenant de la région antrale $(n=42)$ ou du duodénum chez l'homme $(n=9)$. 
avons aussi examiné si la motiline pouvait avoir quelque effet dans la stimulation colique observée après un repas. Malheureusement, la stimulation engendrée par la motiline dans le colon apparaît très faible en période de jeûne et nulle en période postprandiale [15]. Le rôle de la motiline dans la régulation de l'activité digestive postprandiale chez l'homme demeure donc encore incertain. Dans ces conditions l'utilisation d'antagonistes spécifiques de la motiline pourrait s'avérer essentielle pour clarifier les fonctions de ce peptide.

\section{RÉFÉRENCES}

15. Bradette $M$, Riberdy $M$, Raymond $\mathrm{MC}$ et al. Effect of motilin on the contractile activity of the human colon. J Gastroent Motility $1991 ; 3-3: 111$

16. Konturek SJ, Dembinski A, Krol R Wunsch E. Effects of motilin on gastric and pancreatic secretion in dogs. Scand J Gastroent 1976 ; 11(39) : 57-61.

17. Poitras P, Trudel L, Lahaie RG, StPierre S. Stimulation of duodenal muscle contraction by porcine or canine motilin in the dog in vivo. Clin Invest Med $1990 ; 13$, 11-6.

18. Fox-Threlfeld JET, Manaka H, Manaka Y, Cipris S, Woskowska Z, Daniel EE. Mechanism of noncholinergic excitation of canine ileal circular muscle by motilin. Peptides 1991; 12 : 1047-50.

19. Peeters TL, Bormans V, Vantrappen G. Comparison of motilin binding to crude homogenates of human and canine GI smooth muscle tissue. Reg Peptides 1988 ; $23: 171-82$

20. Peeters TL, Matthijs G, Depoortere I Cachet T, Hoogmartens J, Vantrappen G Erythromycin is a motilin receptor agonist . Am J Physiol. 1989 ; 257 : G470-G4.

21. Poitras P, Steinbach JH, Van Deventer G, Code CF, Walsh JH. Motilinindependent ectopic fronts of the interdigestive myalectric complex in dogs. $A m J$ Physiol 1980 ; 239 : G215-G20.

22. Boivin $\mathbf{M}$, Raymond MC, Riberdy $\mathbf{M}$, Trudel L, St-Pierre S, Poitras P. Plasma motilin variation during the interdigestive and digestive states in man. J Gastroint Motility $1990 ; 2$ : 240-6.

23. Lemoyne M, Wassef R, Tassé D, Trudel $\mathrm{L}$ and Poitras $\mathrm{P}$. Motilin and the vagus in dog. Can J Physiol Pharmacol 1984; 62 : 1092-6.

24. Poitras $\mathbf{P}$, Tassé D, Laprise P. Stimulation of motilin release by bombesin in dogs. Am J Physiol 1983 ; 245, G249-G56.

\section{Intérêt clinique}

Étant donné l'activité biologique de la motiline, on pourrait s'attendre à ce qu'une hypermotilinémie s'accompagne d'une hyperactivité de la motricité digestive, et qu'une hypomotilinémie engendre une hypomotricité digestive. Une hypermotilinémie a été retrouvée chez des sujets porteurs de gastrinome, de phéochromocytome, de vipome, de syndrome carcinoïde ou de carcinome pulmonaire à petites cellules [35]. Cependant aucun des patients considérés ne

Trudel L. Regulation of plasma motilin by opioids in the dog. Am J Physiol 1989;257 G41-G5.

26. Poitras P, Dumont A, Cuber JC, Trudel L. Cholinergic regulation of motilin release from isolated canine intestinal cells. Peptides 1993 (in press).

27. Boivin $\mathbf{M}$, Bradette $\mathbf{M}$, Riberdy $\mathbf{M}$, Raymond MC, Poitras P. Mechanisms for motilin release after a meal in humans. Dig Dis Sci 1992 ; 37 : 1562-8.

28. Farrell JI, Ivy AC. Studies on the motility of the transplanted gastric pouch. $A m$ J Physiol 1926 ; 76 : 227-8.

29. Poitras P. Motilin is a digestive hormone in the dog. Gastranterology $1984 ; 87$ 909-13

30. Lee KY, Shiratori K, Chen YF, Chang T, Chey WY. A hormonal mechanism for the interdigestive pancreatic secretion in dogs. Am J Physiol 1986; 251 : G759-G64.

31. Itoh Z, Nakaya M, Suzuki T, Arai H. Wakabayashi K Erythromycin mimics exogenous motilin in gastrointestinal contractile activity in the dog. $A m J$ Physiol $1989 ; 247$ : G688-G94.

32. Tomomasa T, Kuroume T, Arai H Wakabayashi K, Itoh Z Erythromycin induces migrating motor complex in human gastrointestinal tract. Dig Dis Sci $1986 ; 31$ : 157-61.

33. Boivin M, Riberdy $\mathbf{M}$, Raymond MC, Trudel L, Poitras P. Motilin and the postprandial motility of the antrum. Can $J$ Physiol Pharmacol 1992 (in press).

34. Janssens J, Peeters TL, Vantrappen G, Tack J, Urbain JL, DeRoo M, Muls E, Bouillon $R$. Improvement of gastric emptying in diabetic gastroparesis by erythromycin. NEJM 1990 ; 322 : 1028-31.

35. Poitras P. Motilin. In Gut Peptides : Physiology, Biochemistry and Pathophysiology (G. Dockray and J.H. Walsh, eds.), Raven Press (in press). présentait de tableau clinique qu'on a pu associer à l'hypermotilinémie. On a observé également des altérations de la motilinémie chez des patients présentant de la constipation, de la gastroparésie ou d'autres déréglements moteurs intestinaux mais sans qu'on puisse là non plus en tirer de conclusions valables [35].

Suite aux observations récentes démontrant que les dérivés de l'érythromycine pouvaient agir au niveau des récepteurs à la motiline [20] et qu'une activation des récepteurs à la motiline pouvait être obtenue en période postprandiale [32, 33], il existe un indiscutable intérêt thérapeutique en faveur de la motiline. En effet, la stimulation par l'érythromycine de l'activité motrice postprandiale, d'après les expériences réalisées par exemple chez les diabétiques atteints de gastroparésie [34], est supérieure à celle obtenue par l'intermédiaire de n'importe quel autre agent prokinétique jamais testé à ce jour. Ces résultats laissent prévoir que dans un très proche avenir la stimulation des récepteurs gastriques, et/ou duodénaux de la motiline par des dérivés de l'érythromycine, ou par des analogues de la motiline, s'avérerait une option thérapeutique des plus efficaces pour les malades atteints de dyskinésie du tractus digestif

\section{Summary}

Motilin. From animal to man.

Motilin is one of the numerous peptides found in intestinal mucosa. In animals, plasma motilin variations were associated with the regulation of the migrating motor complex. The place of motilin in human physiology has still to be clarified but its importance for the clinician is gradualy emerging. Stimulation of motilin receptors present in the human antrum elicits most potent contractions of this region of the stomach and motilin (or a derivative of motilin) could in the near future constitute the most efficient regimen for the treatment of gastroparesis.

\section{TIRÉS A PART}

P. Poitras. 